\title{
Femoral Nerve Palsy Following Landmark Based Ilioinguinal-Iliohypogastric Nerve Block: Case Report and Safety Review
}

\author{
Logan Thomas Mellert ${ }^{\mathrm{a}}$, , Maureen Elizabeth Cheunga, \\ Rick Alan Gemma ${ }^{a}$
}

\begin{abstract}
Inguinal hernia repair is one of the most commonly performed procedures in the United States. Ilioinguinal-iliohypogastric nerve blocks are commonly performed for postoperative pain control. Transient femoral nerve palsy is a recognized complication of this block. A 75-year-old male underwent an uncomplicated Lichtenstein repair of an incarcerated right inguinal hernia. An ilioinguinal-iliohypogastric nerve block was performed at the conclusion. Postoperatively, he reported symptomatology of femoral nerve palsy, with full resolution within 8 h. Femoral nerve palsy occurs when anesthetic is infiltrated into the transverse abdominis muscle, allowing for diffusion toward the femoral nerve along contiguous planes of the transverse abdominis-iliacus fascia. Incidence can be as high as 5\%. Literature favors ultrasound to landmark-based approaches for improved efficacy and reduced complications. Postoperative protocols for femoral nerve palsy can be implemented with minimal effect on nursing workflow to improve patient safety from the effects of this complication.
\end{abstract}

Keywords: Femoral nerve palsy; Ilioinguinal-iliohypogastric nerve block; Inguinal hernia repair

\section{Introduction}

Inguinal hernia repair is one of the most commonly performed procedures in the United States, with over 800,000 procedures performed annually [1]. Ilioinguinal-iliohypogastric nerve blocks (II/IH-NB) are an important component of perioperative pain management. The II/IH nerves reside between the transversus abdominis and internal oblique muscles entering this plane medial to the anterior superior iliac spine (ASIS) [2]. Cadaveric studies suggest that the transversalis and iliacus

Manuscript accepted for publication April 18, 2017

${ }^{a}$ Western Reserve Hospital, 1900 23rd St, Cuyahoga Falls, OH 44223, USA bCorresponding Author: Logan Thomas Mellert, Western Reserve Hospital, 1900 23rd St, Cuyahoga Falls, OH 44223, USA.

Email:LMELLERT@WesternReserveHospital.org

doi: https://doi.org/10.14740/jmc2809w fascia are contiguous with one another. The continuity of this fascia allows a pathway for anesthetic to track toward the femoral nerve if infiltration occurs beneath or into the transversus abdominis muscle [2]. The result is self-limited femoral nerve palsy (FNP) secondary to anesthetic blockade [3]. Symptomatology of this palsy includes quadriceps muscle weakness, numbness or paresthesias in the anterior thigh, and proximal psoas muscle weakness [4]. We present a case of FNP following intraoperative landmark-based II/IH-NB.

\section{Case Report}

A 75-year-old male presented to a community hospital for elective repair of a chronically incarcerated right inguinal hernia. He reported a 6-year history of incarceration and elects now for repair secondary to intractable discomfort which is lifestyle limiting. He reported no change in hernia size, bowel or bladder habits. A Lichtenstein herniorrhaphy was performed without complication. At the conclusion of the procedure, an II/IH-NB was performed utilizing $10 \mathrm{cc}$ of $0.5 \%$ bupivacaine. In recovery, the patient reported numbness in the right anterior thigh. Upon examination, hip flexor weakness, quadriceps paralysis, and an inability to ambulate were noted. The patient was admitted for observation and had full return of neurologic function $8 \mathrm{~h}$ postoperatively. It was determined that he suffered from transient FNP due to his II/IH-NB. He reported no residual neurologic symptoms at follow-up appointments.

\section{Discussion}

Perioperative pain control is an important component of surgical care. Recently, there has been an increased emphasis on adequate pain management with minimal opioid administration [5-10]. This is particularly important with an aging population who are prone to the adverse effects of opioids including somnolence, cognitive impairment, nausea, vomiting, and respiratory depression [11]. As a result, $\mathrm{II} / \mathrm{IH}$ and transverse abdominis plane (TAP) blocks have gained popularity for perioperative pain control. While the overall safety of these blocks is well established, it is important to be aware of potential complications. 


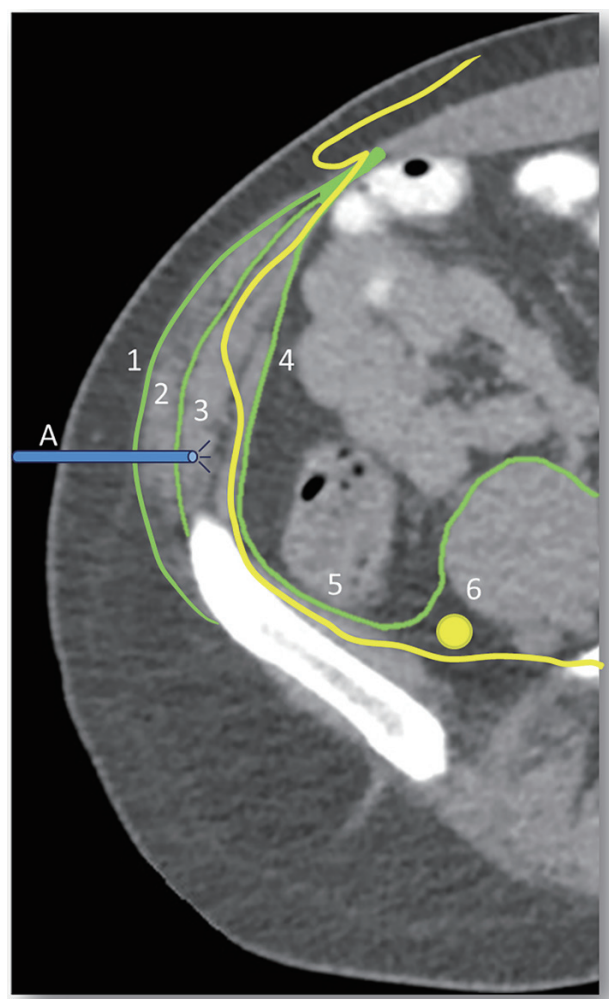

Figure 1. Relevant planes and structures of II/IH blocks. 1: external oblique aponeurosis; 2: internal oblique aponeurosis; 3: $\mathrm{II} / \mathrm{IH}$ nerve penetrating transversus abdominis; 4: transversalis fascia; 5 : iliacus fascia; 6: femoral nerve; A: desired needle depth for II/IH block.

Complications following II/IH-NB are 4.5-9\% [3, 9, 12] with serious complications rarely reported. In addition to transient FNP, complications include trauma to blood vessels, pelvic hematoma, intravascular injection, urinary retention, and bladder/enteric penetration $[3,5,9,12]$. Practitioner experience and modality of administration have been associated with complications and failure of NB to provide adequate analgesia $[5,13]$. In an effort to avoid these complications, ultrasoundguidance was introduced as an alternative to traditional landmark-based administration.

Conventional landmark-based infiltration, known as a "blind" technique, utilizes the ASIS as its primary landmark. While all sources agree that the insertion occurs $2 \mathrm{~cm}$ medial to the ASIS, there is variation in coronal infiltration from $2 \mathrm{~cm}$ superior/inferior to the ASIS $[7,13]$. This variation is in part due to anatomic variations of the II/IH nerves. In a study by AlDabbagh, the courses were identified in 110 primary inguinal hernia repairs and compared to accepted anatomic texts. They were consistent in only $41.8 \%$ of cases [14]. The appropriate depth of penetration is determined by two palpable "clicks" as the needles transverses of the external and internal oblique aponeurosis (Fig. 1).

Local anesthetic, typically $0.5 \%$ bupivacaine, is infiltrated into this plane $[2,13]$. Inadequate analgesic rates of $28-45 \%$ are reported $[5,7,13]$. Suggested mechanisms include unpredictable nerve course, inaccuracy of tactile clicks, user experience and anatomic muscular variation [5]. The combined

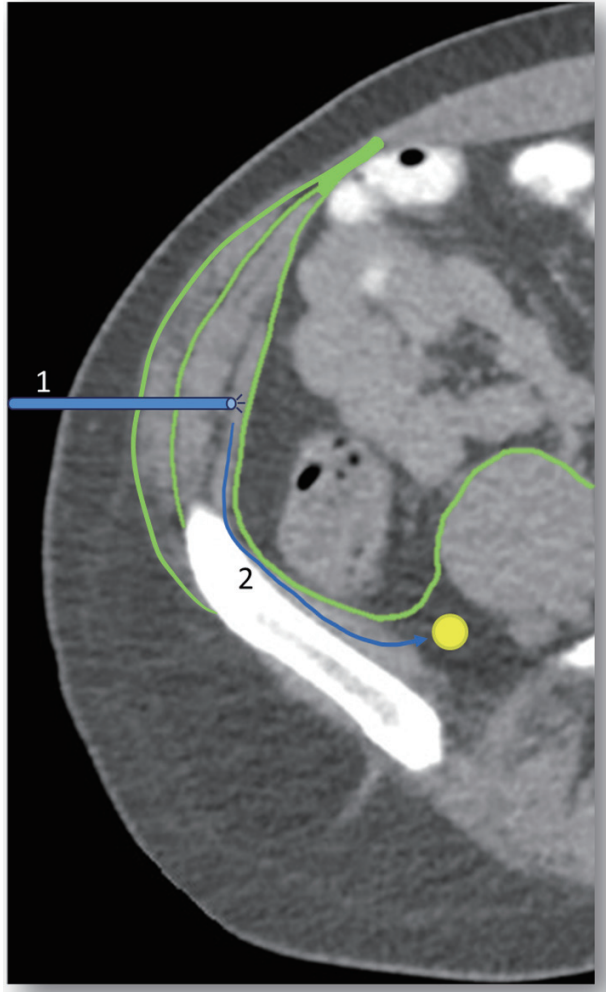

Figure 2. Improper administration depth. 1: Improper penetration through, into, or deep to the transversalis muscle. 2: As the transversalis and iliacus fascia are contiguous with one another, infiltration into this plane can result in tracking, inadvertently anesthetizing the femoral nerve.

thickness of the internal oblique and transversus abdominis muscles, which constitutes the desired landing zone, is less than $5 \mathrm{~mm}$ in cadaveric studies [2]. Previously mentioned complications are secondary to inappropriate infiltration depth into or through the transversus abdominis muscle (Fig. 2).

Several adjustments to the basic technique have been utilized in an attempt to produce consistent analgesic results. These include a fan distribution of infiltration, multiple site injection, and direct visualization $[5,7,10,13]$.

Ultrasound (US) guidance is a well-documented technique that allows direct visualization during infiltration [5-7, 13]. In this method, a high frequency probe is placed equidistance between the iliac crest and costal margin. The ilioinguinal and iliohypogastric nerves are then identified between the transversus abdominis and internal oblique muscles. The area surrounding the nerves is infiltrated with local anesthetic until they appear to be completely surrounded with fluid. This characteristic visual effect is referred to as the kayak sign [7]. It is recognized that this anatomic identification can be difficult and proficiency is associated with a learning curve. When performed by an experienced physician, US blocks have rapid onset, lower total dose administration, and more consistent analgesic effect than landmark-based methods [5, 7, 13].

Another method of visualized infiltration is administration during laparoscopic procedures $[15,16]$. This nerve blockade, typically utilized for TAP blocks, is performed during pneu- 
moperitoneum with direct laparoscopic visualization. The needle is introduced at insertion sites in the midaxillary line equidistance between the iliac crest and costal margin. Two distinct "clicks" are felt and analgesic is infiltrated. Laparoscopic views are used to confirm bulging of the transversus abdominis muscle. This method similarly attempts to eliminate potential complications, specifically colonic or small bowel perforation which has been described with the other administration techniques. Proponents also note that there is little to no learning curve with this method which takes advantage of tactile sensation and laparoscopic visualization $[15,16]$.

Though the incidence of FNP following II/IH-NB is an uncommon occurrence, it does represent a potential risk to patient safety. Though there were no significant safety events in this case, there are reports of patient falls resulting in significant injuries including ankle sprains, ankle fractures, and femoral neck fractures $[12,17,18]$. This risk is increased in the elderly, and those with baseline ataxia or neuromuscular disorders. There is no literature discussing postoperative safety screening measures aimed at identifying this complication. As a result of this occurrence, the institution performed a review for FNP screening following II/IH and TAP-NB. Screening had to be low cost, with minimal effect on nursing workflow. Patients receiving NB undergo a focused neurologic exam of the lower extremities by the nursing staff prior to ambulation and discharge home. Motor examination with strength testing of the hip and knee flexion/extension is tested. Sensation of the anterior thigh is also assessed. If the patient has no new neurologic deficits, the patient undergoes assisted ambulation. If there are no perceived deficits, the patient follows standard same day surgery discharge criteria. When deficits are noted, the physician is notified and the self-limited nature of this complication is discussed with the patient and responsible party. Final recommendations for observation or discharge are made on a case by case basis pending function status, debility, and home support. This protocol can be effectively implemented and adopted by recovery nurses with minimal effect to current workflow. This ensures safe and appropriate disposition of the patient, minimizing the potential risk of falling.

\section{Conclusions}

Inguinal hernia repairs are one of the most commonly performed surgical procedures. The integration of regional anesthetic NBs has decreased the opiod administration following these surgeries. Application of US and laparoscopic guidance has improved the safety and efficacy of these blockades. While this has proved an effective method of perioperative analgesia, it also introduces a new complement of complications. Transient FNP is one of these complications and is of particular interest due to its potential for significant patient injury if it is not promptly recognized. With identification, simple safety care plans can be developed on an individual basis. In this case, serious safety events were avoided. This has led to the integration of a new postoperative safety assessment aimed at prompt identification of FNP and appropriate disposition of those patients.

\section{Grants}

None.

\section{Financial Disclosures}

None.

\section{Conflicts of Interest}

None.

\section{Abbreviations}

II-IH: ilioinguinal-iliohypogastric nerve; FNP: femoral nerve palsy; NB: nerve block; ASIS: anterior superior iliac spine; TAP: transverse abdominis plane; US: ultrasound

\section{References}

1. Rutkow IM. Demographic and socioeconomic aspects of hernia repair in the United States in 2003. Surg Clin North Am. 2003;83(5):1045-1051, v-vi.

2. Rosario DJ, Jacob S, Luntley J, Skinner PP, Raftery AT. Mechanism of femoral nerve palsy complicating percutaneous ilioinguinal field block. Br J Anaesth. 1997;78(3):314-316.

3. Larson S, Laird R, Brody F. Femoral Nerve Paralysis Following Open Inguinal Hernia Repair. Am J Med Case Rep. 2015;3(3):85-87.

4. DeLee J, Drez D, Miller MD, eds. DeLee \& Drez's Orthopaedic Sports Medicine: Principles and Practice. 3rd ed. Philadelphia: Saunders/Elsevier; 2010.

5. Wang Y, Wu T, Terry MJ, Eldrige JS, Tong Q, Erwin PJ, Wang Z, et al. Improved perioperative analgesia with ultrasound-guided ilioinguinal/iliohypogastric nerve or transversus abdominis plane block for open inguinal surgery: a systematic review and meta-analysis of randomized controlled trials. J Phys Ther Sci. 2016;28(3):1055-1060.

6. Stav A, Reytman L, Stav MY, Troitsa A, Kirshon M, Alfici R, Dudkiewicz M, et al. Transversus Abdominis Plane Versus Ilioinguinal and Iliohypogastric Nerve Blocks for Analgesia Following Open Inguinal Herniorrhaphy. Rambam Maimonides Med J. 2016;7(3).

7. Khedkar SM, Bhalerao PM, Yemul-Golhar SR, Kelkar $\mathrm{KV}$. Ultrasound-guided ilioinguinal and iliohypogastric nerve block, a comparison with the conventional technique: An observational study. Saudi J Anaesth. 2015;9(3):293-297.

8. Crompton JG, Dawes AJ, Donald GW, Livhits MJ, Chandler CF. Perineural bupivacaine injection reduces inguinodynia after inguinal hernia repair. Surgery. 2016;160(6):1528-1532.

9. Shivashanmugam T, Kundra P, Sudhakar S. Iliac com- 
partment block following ilioinguinal iliohypogastric nerve block. Paediatr Anaesth. 2006;16(10):1084-1086.

10. Toivonen J, Permi J, Rosenberg PH. Analgesia and discharge following preincisional ilioinguinal and iliohypogastric nerve block combined with general or spinal anaesthesia for inguinal herniorrhaphy. Acta Anaesthesiol Scand. 2004;48(4):480-485.

11. Chau DL, Walker V, Pai L, Cho LM. Opiates and elderly: use and side effects. Clin Interv Aging. 2008;3(2):273-278.

12. Manatakis DK, Stamos N, Agalianos C, Karvelis MA, Gkiaourakis M, Davides D. Transient femoral nerve palsy complicating "blind" transversus abdominis plane block. Case Rep Anesthesiol. 2013;2013:874215.

13. Trainor D, Moeschler S, Pingree M, Hoelzer B, Wang Z, Mauck W, Qu W. Landmark-based versus ultrasoundguided ilioinguinal/iliohypogastric nerve blocks in the treatment of chronic postherniorrhaphy groin pain: a retrospective study. J Pain Res. 2015;8:767-770.
14. al-dabbagh AK. Anatomical variations of the inguinal nerves and risks of injury in 110 hernia repairs. Surg Radiol Anat. 2002;24(2):102-107.

15. Favuzza J, Delaney CP. Laparoscopic-guided transversus abdominis plane block for colorectal surgery. Dis Colon Rectum. 2013;56(3):389-391.

16. Keller DS, Ermlich BO, Schiltz N, Champagne BJ, Reynolds HL, Jr., Stein SL, Delaney CP. The effect of transversus abdominis plane blocks on postoperative pain in laparoscopic colorectal surgery: a prospective, randomized, double-blind trial. Dis Colon Rectum. 2014;57(11):12901297.

17. Walker G. Transversus abdominis plane block: a note of caution! Br J Anaesth. 2010;104(2):265.

18. Callesen T, Bech K, Kehlet H. One-thousand consecutive inguinal hernia repairs under unmonitored local anesthesia. Anesth Analg. 2001;93(6):1373-1376, table of contents. 